\title{
Feasibility and efficacy of the Vojta therapy in subacromial impingement syndrome: a randomized controlled trial
}

\author{
María Lidia Juárez-Albuixech', Olga Redondo-González², Inmaculada Tello-Díaz-Maroto³, Jorge Lucas Torres de la Guía4, \\ Jorge Hugo Villafañ e, ${ }^{5, *}$ Carmen Jiménez-Antona ${ }^{6}$
}

${ }^{1}$ Servicio de Rehabilitación. Hospital Universitario doctor Peset. Valencia, Spain

${ }^{2}$ Preventive Medicine and Public Health Sevice, University Hospital of Guadalajara, Guadalajara, Spain

${ }^{3}$ Autónoma University, Madrid, Spain

${ }^{4}$ Ouintanar de la Orden Health Centre, Toledo, Spain

${ }^{5}$ RCCS Fondazione Don Carlo Gnocchi, Milan, Italy

${ }^{6}$ Rey Juan Carlos University, Madrid, Spain

Subacromial impingement syndrome (IS) is a common disorder associated with functional impairment and disability of the shoulder. The aim of this study was to evaluate the efficacy of Vojta therapy (VT) techniques compared with standard therapy (ST) in patients with subacromial IS. A randomized controlled trial was performed, with 60 patients with IS (mean age, $61 \pm 12$ years) randomized into two groups: $S T(n=30)$ and ST plus Vojta therapy (SVT, $n=30$ ). Both received 15 treatment sessions over 4 weeks. Outcome measures included pain intensity, functionality, joint range of motion (RoM), strength, and quality of life measurements. The efficacy of both treatments was analysed, both com- paratively and separately, immediately after completion of treatment and after 3 months. After the intervention, both groups showed statistically significant differences in visual analogue scale $(P=0.001)$, RoM $(P<0.01)$, and strength $(P<0.001)$, which were also seen 3 months after the intervention. SVT is more efficient in both the short and medium term in reducing pain, improving functionality, increasing articular RoM and strength, and offering a better quality of life in IS patients.

Keywords: Subacromial impingement syndrome, Pain, Vojta therapy, Musculoskeletal disorder

\section{INTRODUCTION}

Subacromial impingement syndrome (IS) consists of an irritation of the rotator cuff tendons where they pass through the coracoacromial arch during shoulder elevation. This causes a narrowing acromiohumeral distance, which is thought to be the cause of subacromial IS-related pain in the anterolateral and upper shoulder, frequently reducing its functionality, mainly with regard to abduction, rotation, and flexion of over $60^{\circ}$ (Ishii et al., 2021; Michener et al., 2004).

Patient ages (range, 42-58 years) are typical for IS (Kuhn, 2009). The prevalence of shoulder pain is reported to range between $7 \%$ and $27 \%$ in the general population and can be even higher in athletic populations with frequent overhead arm activity, where it can range from $36 \%$ to $66 \%$. Shoulder pain has an incidence of 9-25 cases per 103 population/yr (Villafane et al., 2019). Indeed, IS is the most frequent alteration of the shoulder's articular complex of diverse aetiology (Calis et al., 2011). Due to the pain, $40 \%$ to $50 \%$ of those affected seek medical attention; the pain persists 12 months after the first medical consultation in half of these cases (van der Windt et al., 1996), causing a lack of productivity and a considerable drain on healthcare resources (Bongers, 2001).

Initial treatment of IS is conservative in most cases (Varela et al., 2013). If the initial treatment fails, arthroscopy may be recommended for decompression (Guyette et al., 2002). The standard treatment (ST) applied in the physiotherapy unit (UF) is prescribed by a rehabilitation specialist, consisting of one or more of the following procedures: transcutaneous electrical nerve stimulation
${ }^{*}$ Corresponding author: Jorge Hugo Villafañe (iD https://orcid.org/0000-0002-3239-7626 IRCCS Fondazione Don Carlo Gnocchi, Piazzale Morandi 6, Milan 20121, Italy Email: mail@villafane.it

Received: June 5, 2021 / Accepted: July 9, 2021
This is an Open Access article distributed under the terms of the Creative Commons Attribution Non-Commercial License (https://creativecommons.org/licenses/by-nc/4.0/) which permits unrestricted non-commercial use, distribution, and reproduction in any medium, provided the original work is properly cited. 
(TENS), ultrasound therapy, kinesiotherapy, and cryotherapy (Taveggia et al., 2016).

Reflex locomotion or Vojta therapy (VT) consists of administering pressure to defined zones of the body with the patient in the prone, supine, or side-lying position to activate global and innate locomotive patterns or complexes, namely the creeping reflex and the rolling reflex. Both complexes provoke coordinated, rhythmic activation of the skeletal musculature throughout the entire body (Juarez-Albuixech et al., 2020). This enables a change from pathological patterns to alternative physiological patterns that are painless, efficient, and functional, by means of generating significant global effects, including the axial extension of the spine, correct positioning of the shoulder girdle, and activation of the abdominal musculature (Phillips, 2014), all of which are altered by shoulder pathologies. We hypothesise that VT is a safe and efficient conservative alternative. This new possibility, which would be accessible to any physiotherapeutic facility, could have a considerable economic and social impact, considering that it sustains and even improves patient functionality over time. Our objective was to examine the effectiveness of a VT and standard approach relative to a standard intervention in patients with subacromial IS.

\section{MATERIALS AND METHODS}

\section{Study design}

This is an analytical experimental study conducted as a random- ized clinical trial with two parallel intervention levels: a control group and an experimental group. All patients were diagnosed with IS. All participants read and signed an informed consent form prior to study enrolment. The study protocol was approved by the local Ethical Committee of the Mancha Central Hospital and conducted in accordance with the Helsinki declaration. This project was retrospectively registered in ClinicalTrials.gov with the number NCT04102397 in September 25, 2019. The assignation process for either the standard or experimental therapy, as well as the subjects who did not participate in the final study are shown in Fig. 1.

\section{Participants}

Sixty-five potentially eligible patients were recruited from IS associations from February 2015 through December 2016. Inclusion criteria for this study were: Patients aged between 18 and 85 years and diagnosed previously with IS, who fulfil at least three of the following criteria: (a) pain in active abduction $\left(70^{\circ}-120^{\circ}\right.$ ), considered impingement + ; (b) pain on palpation of any of the following osteotendinous insertions: infraspinatus, teres minor, supraspinatus, subscapularis, and biceps; (c) pain in any of the following isometric contractions: abduction (first $10^{\circ}$ ), internal rotation (in the anatomical position and with $90^{\circ}$ elbow flexion), and shoulder flexion; (d) positive on the Neer and Hawkins tests (Phillips, 2014); or (e) night-time shoulder pain. Patients were excluded from enrolment if they had signs of a full-thickness rotator cuff tear; acute inflammation or cervical radicular pain; calcification of

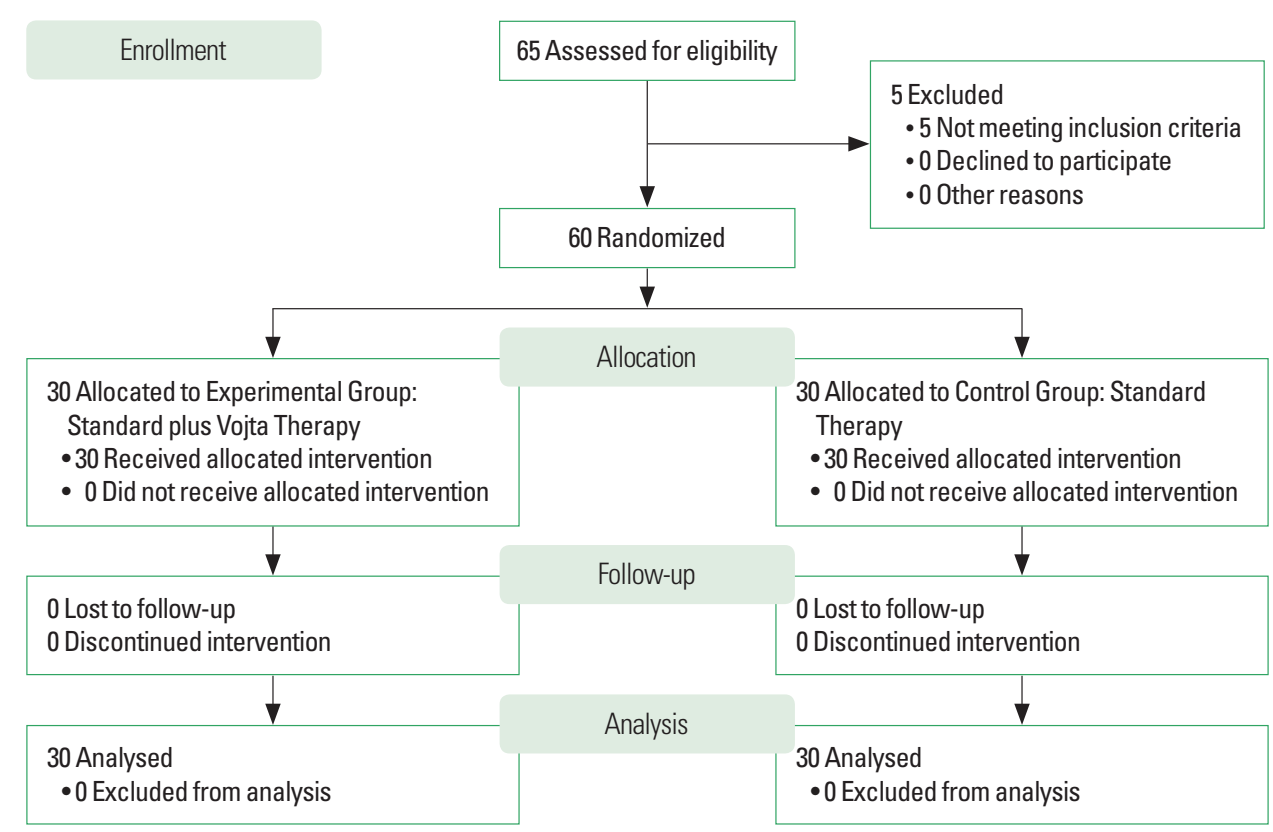

Fig. 1. Consort flow chart. 
the cuff tendons; glenohumeral instability; previous shoulder surgery; limited passive joint balance; pain due to suspected visceral or infectious process; shoulder pain of neurological origin; bilateral involvement; physiotherapeutic treatment in the past 6 months; aged $<18$; or physical and/or psychological dependence.

\section{Randomization and blinding}

The randomization sequence was generated using the programme EPIDAT ver. 4.2 (Xunta de Galicia, Galicia, Spain). The sequence consisted of random permutation blocks, each with four to six elements of length, to avoid imbalances in the groups in case of attrition. The sequence was concealed in sealed, serialised, opaque envelopes (Schulz et al., 1995). The necessary sample size was calculated taking into account the number of samples required for the main result indicator (level of pain). We expected to find an improvement of at least 2 points with the implementation of VT. We therefore assumed a variability of 2.5 points (standard deviation) on the pain scale, a value previously estimated in patients suffering shoulder pain in the same healthcare area. In order to detect this difference with a power of $80 \%(\beta$ error $=20 \%)$ and a $95 \%$ confidence interval $(\mathrm{CI})(\alpha$ error $=5 \%)$, it was necessary to recruit 25 patients per group.

\section{Interventions}

The patients that fulfilled all inclusion criteria and gave their informed consent to participate were assigned either to the control group or to the experimental group at random and with equal probability. Interventions in both groups were applied by the same physical therapist with 3-year experience in manual therapy and the management of musculoskeletal pain disorders. Both groups were provided with the same instructions regarding effectiveness of the treatment.

(a) Control group: standard therapy (ST) $(\sim 50 \mathrm{~min} / \mathrm{session})$ :

- TENS $(20 \mathrm{~min}$, phase duration $=0.10 \mu \mathrm{sec}$, frequency = $50 \mathrm{~Hz}$ ). The intensity applied depended on the patient's tolerance for the treatment (Fig. 2A). Ultrasound (5 min, frequency $=1 \mathrm{MHz}$, intensity $\left.=1 \mathrm{~W} / \mathrm{cm}^{2}\right)$ (Fig. 2B).

- Kinesiotherapy. In stages: at first assisted (with isometric exercises), then free, and finally, with resistance, usually using elastic bands (Fig. 2C).

- Cryotherapy (5 min).

(b) Experimental group: standard plus VT (SVT) (VT, 20 min/ session): application of the reflex creeping complex, activating several stimulus points on the facial and the nuchal sides (Fig. 3A), and then activation of the reflex rolling complex
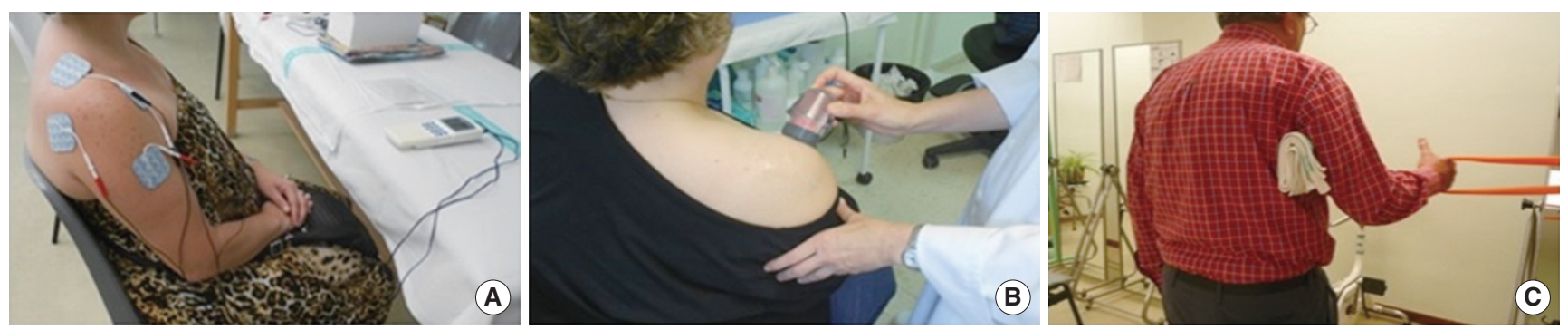

Fig. 2. Control group. (A) Application of transcutaneous electrical nerve stimulation current. Placement of four electrodes on the skin of the areas identified as the most painful (normally under the acromion and above the supraspinatus. Application of cathodes at the most painful points, and of the anodes near them. (B) Application of pulsating ultrasound. (C) Rehabilitation through kinesitherapy.
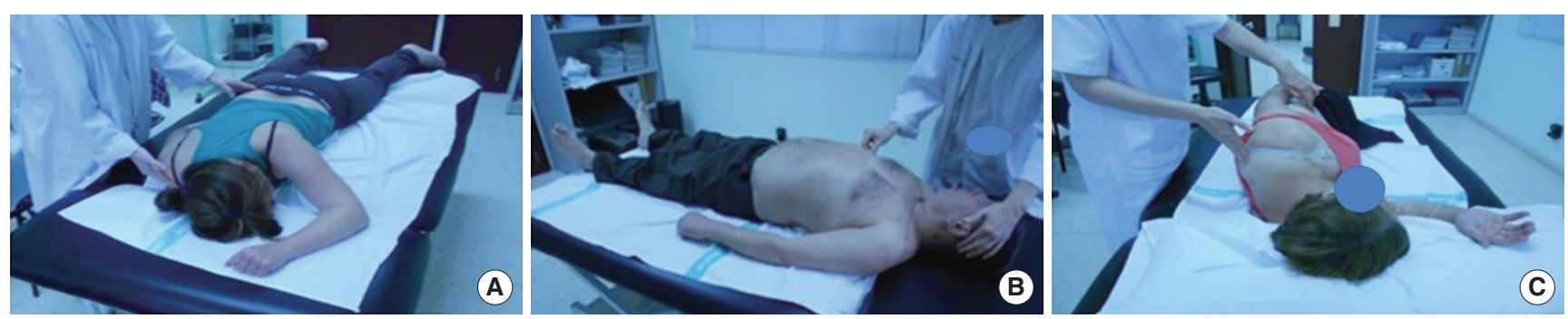

Fig. 3. Experimental group. (A) Crawling reflex. Stimulation of the acromial area and the buttock on the nuchal side. (B) Rolling reflex, supine position. Stimulation of the pectoral area with head-guided resistance. (C) Rolling reflex, lateral position. Areas of stimulation: medial edge of the scapula and anterior superior iliac spine from the top side. 
in both the supine position and with the patient lying on their side (Fig. 3B, C), alternating between nuchal and facial stimulation and guiding from the head.

\section{Outcome measures}

Changes in the following variables; pain (main result indicator), RoM, strength, functionality; and quality of life (QoL), were analysed using validated measurement scales. Pain intensity was assessed using a visual analogue scale $(\mathrm{VAS}=0$ : no pain, and VAS= 10: maximum pain) (Hjermstad et al., 2011). The Disabilities of the Arm, Shoulder, and Hand (DASH) Questionnaire was used, which is the most commonly used questionnaire to globally evaluate the functionality of the upper limbs from the patient's perspective. It has excellent reproducibility, high sensibility, good internal consistency, and a high sensitivity to changes (Hervas et al., 2006). The Constant-Murley Scale (CMS) was also used, which is an evaluation system of shoulder functionality more commonly used in Europe and the United States. It assesses pain, functionality, strength, and joint RoM (Constant et al., 2008). QoL was assessed using the 12-item Short Form (SF-12) Health Survey. A shortened version of the 36-item Short Form, this survey has a high internal consistency, validity, and reliability. It is the most widely used health measurement in the medical field. Using 12 categories, it evaluates eight positive and negative dimensions of physical and mental health, and, by extension, of QoL (Schulz et al., 1995). These questionnaires were given to each patient three times: after signing the informed consent form, at the end of the 15 therapy sessions, and 12 weeks after the start of the therapy. An assessor blinded to the patients' allocated group obtained measurements at baseline, immediately after the 15 -session treatment period, and at 3 months after the treatment.

\section{Statistical analysis}

Data were analysed using IBM SPSS Statistics ver. 22.0 (IBM Co., Armonk, NY, USA), with an intention-to-treat analysis using the last-value-forward method. The results are expressed as means, standard deviations, and/or 95\% CIs. The Kolmogorov-Smirnov test showed a normal distribution of the data. Separate $2 \times 3$ mixed model analysis of variances, with the group (experimental, control) as the between-subjects factor and time (baseline, postintervention at 4 weeks, and 3-month follow-up) as within-subject factors, were conducted to examine the effects of the intervention on VAS, DASH, and CMS as the dependent variables. The main hypothesis of interest was the group $\times$ time interaction. post hoc comparisons were conducted with Bonferroni correction. Between- groups effect sizes were calculated using Cohen $d$ coefficient. We considered an effect size greater than 0.8 large, around 0.5 moderate, and less than 0.2 small (Citrome and Magnusson, 2014). The statistical analysis was conducted at a 95\% CI and $P<0.05$ was considered statistically significant.

\section{RESULTS}

\section{Patient demographic and clinical data}

Sixty-five consecutive patients were screened and 60 patients (61 \pm 12 years, $70 \%$ women) were eligible and agreed to participate. Fig. 1 shows the recruitment and retention of patients throughout the trial. Baseline characteristics of the patients in each group are presented in Table 1. No significant differences were found between the characteristics of the two treatment groups.

\section{Pain intensity}

The time factor $(F=146.312 ; P<0.001)$ and the group $\times$ time interaction $(F=61.142 ; P<0.001)$ were significant for pain intensity measured by VAS. post hoc analysis indicated that patients with IS receiving the experimental therapy had a significant reduction in pain in the postintervention period (experimental group: mean, 3.9; 95\% CI, 3.2-4.6; control group: mean, 1.0; 95\% CI, $0.3-1.7, P=0.003$; significant difference between groups 1.5; $95 \%$ CI, 0.4-2.5; $P=0.006$ ), as well as at 3-month follow-up (experimental group: mean, 5.3; 95\% CI, 34.6-6.1, $P<0.001$; control group: mean, $1.1 ; 95 \% \mathrm{CI}, 0.4-1.8 ; P=0.001$; difference between groups 2.8 ; $95 \% \mathrm{CI}, 1.7-3.8 ; P<0.001$ ) period (Table 2). Betweengroups effect sizes were large $(d=1.1)$ after the intervention and $(d=1.4)$ at 3-month follow-up.

Table 1. Characteristics of patients at baseline

\begin{tabular}{lccc}
\hline Characteristic & Exp $(\mathrm{N}=30)$ & Con $(\mathrm{N}=30)$ & $P$-value \\
\hline Age $(\mathrm{yr})$ & $58.5 \pm 13$ & $63.5 \pm 10.5$ & 0.105 \\
Weight $(\mathrm{kg})$ & $78.1 \pm 16.2$ & $78.6 \pm 14.3$ & 0.119 \\
Height $(\mathrm{cm})$ & $160.6 \pm 9.6$ & $161 \pm 8.9$ & 0.186 \\
Body mass index $\left(\mathrm{kg} / \mathrm{m}^{2}\right)$ & $30.0 \pm 6.1$ & $30.6 \pm 5.0$ & 0.263 \\
Progression time $(\mathrm{mo})$ & $18.6 \pm 37.3$ & $11.1 \pm 11.3$ & 0.340 \\
Gender & & & \\
Female & $19(63.3)$ & $23(76.7)$ & 0.260 \\
$\quad$ Male & $11(36.7)$ & $7(23.3)$ & \\
Level of education & & & \\
$\quad$ Primary & $25(83.3)$ & $27(90)$ & 0.220 \\
Secondary & $4(13.3)$ & $3(10)$ & \\
Advanced & $1(3.3)$ & - & \\
\hline
\end{tabular}

(Continued to the next page) 
Table 1. Continued

\begin{tabular}{|c|c|c|c|}
\hline Characteristic & $\operatorname{Exp}(N=30)$ & Con $(N=30)$ & $P$-value \\
\hline Employment status & & & 0.609 \\
\hline On leave & $1(3.7)$ & - & \\
\hline Active & $8(25.9)$ & $6(20.7)$ & \\
\hline Retired & $8(25.9)$ & $6(20.7)$ & \\
\hline Homemaker & $11(37.1)$ & $16(51.7)$ & \\
\hline Unemployed & $2(7.4)$ & $2(6.9)$ & \\
\hline Type of work & & & 0.519 \\
\hline UL mobility & $23(76.7)$ & $25(83.3)$ & \\
\hline UL sedentary & $7(23.3)$ & $5(16.7)$ & \\
\hline Affected limb & & & 0.301 \\
\hline Left & 16 (53.3) & $12(40)$ & \\
\hline Right & $14(46.7)$ & $18(60)$ & \\
\hline Dominant limb & & & 0.196 \\
\hline Yes & $12(40)$ & $17(56.7)$ & \\
\hline No & $18(60)$ & $13(43.3)$ & \\
\hline Analgesics & & & 0.999 \\
\hline Yes & $15(50)$ & $16(53.3)$ & \\
\hline No & $15(50)$ & $14(46.7)$ & \\
\hline Previous treatments & & & 0.467 \\
\hline None & $17(56.7)$ & $15(50)$ & \\
\hline Physiotherapy & $5(16.7)$ & $6(20)$ & \\
\hline Drugs & $4(13.3)$ & $7(23.3)$ & \\
\hline Drugs and physio & $4(13.3)$ & $2(6.7)$ & \\
\hline Sedentary habits & & & 0.999 \\
\hline Yes & $4(13.3)$ & $3(10)$ & \\
\hline No & $26(86.7)$ & $27(90)$ & \\
\hline No. of CV risk factors & & & 0.237 \\
\hline 0 & $19(63.3)$ & $16(53.3)$ & \\
\hline 1 & $7(23.3)$ & $10(33.3)$ & \\
\hline 2 & $4(13.3)$ & $2(6.7)$ & \\
\hline 3 & - & $2(6.7)$ & \\
\hline \multicolumn{4}{|l|}{ Tobacco use } \\
\hline Yes & $4(13.3)$ & $2(6.7)$ & \\
\hline No & $26(86.7)$ & 28 (93.3) & \\
\hline Hypothyroidism & & & 0.492 \\
\hline Yes & $2(6.7)$ & - & \\
\hline No & 28 (93.3) & $30(100)$ & \\
\hline Mood disorders & & & 0.999 \\
\hline Yes & $3(10)$ & $4(13.3)$ & \\
\hline No & $27(90)$ & $26(86.7)$ & \\
\hline Neuromuscular disorders & & & 0.353 \\
\hline Yes & $1(3.3)$ & $4(13.3)$ & \\
\hline No & $29(96.7)$ & $26(86.7)$ & \\
\hline Osteoarticular diseases & & & 0.542 \\
\hline Yes & $24(80)$ & 22 (73.3) & \\
\hline No & $6(20)$ & $8(26.7)$ & \\
\hline
\end{tabular}

Values are presented as mean \pm standard deviation or number $(\%)$. Exp, experimental group; Con, control group; UL, upper limb; CV, cardiovascular.

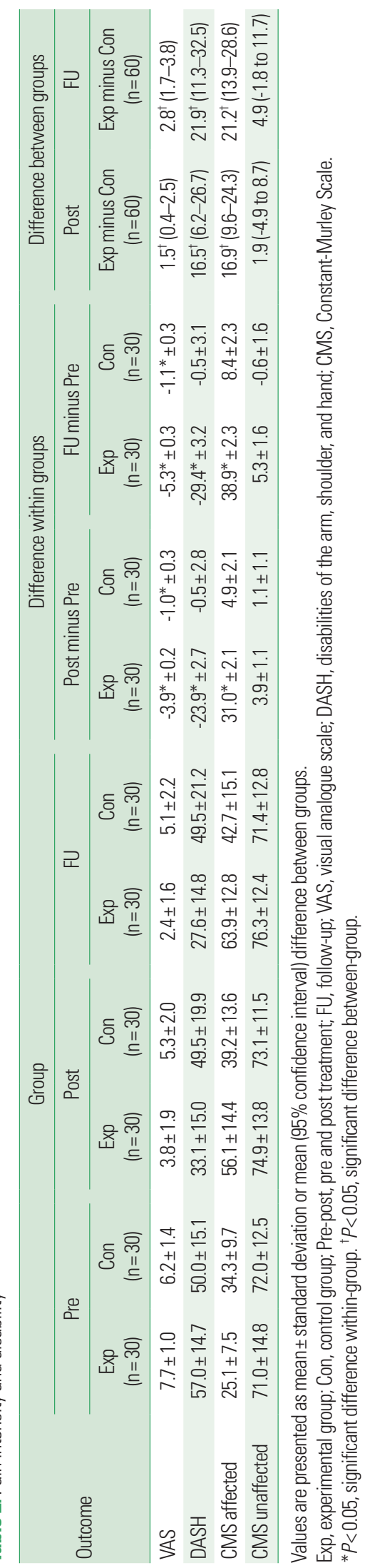




\section{Disability}

The outcome for DASH, RoM, and strength of the affected limb (CMS) demonstrated a significant time factor $(F=22.937$ and 115.838 , both $P<0.001)$ and a significant group $\times$ time interaction $(F=21.265$ and 52.624 ; both $P<0.003)$, respectively. The post hoc testing revealed significant increases in DASH and CMS postintervention compared with the baseline data (all, $P<0.002$ ), and at 3 -month follow-up (all, $P<0.001$ ), in patients receiving the SVT protocol. There were significant differences between the groups $(P=0.02)$ at postintervention and the 3-month follow-up $(P<0.001)$ (Table 2).

\section{Quality of life}

In terms of QoL, ST led to improvements in two dimensions immediately after treatment: role-physical $(P=0.01)$ and vitality $(P=0.004)$. These improvements persisted 3 months after the con-

Table 3. Comparison of the evolution of joint pain, functionality, strength, and range of motion between the two groups under treatment (standard and experimental) after therapy completion and a 3-month follow-up*

\begin{tabular}{|c|c|c|c|}
\hline Variable & Standard & $t$ & $P$-value \\
\hline VAS basal-final Standard & -0.97 & 7.714 & $<0.0001$ \\
\hline VAS basal-final Standard+Vojta & -3.95 & & \\
\hline VAS basal-3 months Standard & -1.10 & 10.393 & $<0.0001$ \\
\hline VAS basal-3 months Standard+Vojta & -5.34 & & \\
\hline VAS final-3 months Standard & -0.14 & 3.477 & 0.001 \\
\hline VAS final-3 months Standard+Vojta & -1.43 & & \\
\hline DASH basal-final Standard & -0.30 & 6.489 & $<0.0001$ \\
\hline DASH basal-final Standard+Vojta & -24.58 & & \\
\hline DASH basal-3 months Standard & -0.52 & 6.476 & $<0.0001$ \\
\hline DASH basal-3 months Standard+Vojta & -29.41 & & \\
\hline DASH final-3 months Standard & -0.61 & 1.773 & 0.084 \\
\hline DASH final-3 months Standard+Vojta & -5.30 & & \\
\hline CMS affected basal-final Standard & 4.83 & -8.781 & $<0.0001$ \\
\hline CMS affected basal-final Standard+Vojta & 30.87 & & \\
\hline CMS affected basal-3 months Standard & 8.41 & -8.245 & $<0.0001$ \\
\hline CMS affected basal-3 months Standard+Vojta & 38.85 & & \\
\hline CMS affected final-3 months Standard & 3.55 & -0.089 & 0.930 \\
\hline CMS affected final-3 months Standard+Vojta & 7.84 & & \\
\hline CMS unaffected basal-final Standard & 0.99 & -2.280 & 0.029 \\
\hline CMS unaffected basal-final Standard+Vojta & 3.70 & & \\
\hline CMS unaffected basal-3 months Standard & -0.62 & -2.104 & 0.043 \\
\hline CMS unaffected basal-3 months Standard+Vojta & 5.36 & & \\
\hline CMS unaffected final-3 months Standard & -1.65 & -0.672 & 0.506 \\
\hline CMS unaffected final-3 months Standard+Vojta & 1.37 & & \\
\hline
\end{tabular}

VAS, visual analogue scale; DASH, disabilities of the arm, shoulder, and hand; CMS, Constant-Murley Scale.

*Hypothesis testing carried out with Student t-test for independent samples. clusion of treatment ( $P=0.04$ and $P=0.01$, respectively). Patients who received SVT also noted the following changes upon completion of therapy: improved physical functioning $(P=0.01)$; a reduction of bodily pain $(P<0.0001)$; better general health $(P=0.04)$, and improved mental health $(P=0.008)$. When compared to baseline values, the results showed that the changes in physical functioning $(P=0.006)$, bodily pain $(P=0.002)$, and mental health $(P=0.002)$ were maintained after the 3 -month follow-up period (Table 3).

\section{DISCUSSION}

These results indicate that upon completion of either the standard or experimental therapy, as well as after the 3-month follow-up period for either treatment, patients noted improvements in pain, RoM, and strength. However, the results are clearly superior when VT is added to the treatment. Furthermore, improvements in functionality and QoL were only observed in patients receiving VT along with the ST.

Specifically, upon completion of SVT and after the 3-month follow-up, the decrease in pain experienced by patients as measured using the VAS scale was 3.0 and 4.2 points greater, respectively, than that experienced by patients in the control group. The literature contains several studies that have observed reductions in bodily pain at the end of ST according to the VAS scale. The efficacy of different modalities (ultrasound therapy, laser treatments, and therapeutic exercises) (Calis et al., 2011) for treating the pain caused by IS have been assessed, and although the majority of studies have focused on the effects of therapeutic exercises (Holmgren et al., 2012; Lewis, 2012; Litchfield, 2013), all treatment methods have been shown to lead to improvements. In our study, the efficacy of the experimental treatment was not only maintained, but also increased after 3 months.

Indeed, our results from the DASH test show that functionality only improved after SVT, an improvement that was 3.5 points greater after the 3-month follow-up period. Likewise, at the end of the treatment period, changes in the role-physical subdomain of the SF-12 Survey were only observed in the SVT group, with these effects increasing by 17 points at the end of the 3-month follow-up. Other authors have made reference to improvements in functionality according to the DASH scale after applying strengthening exercises (Lewis, 2012); however, these studies contain no short- or long-term follow-up analyses.

We also found that deficits in RoM and strength (as measured using the Constant-Murley Scale) improved in both groups, an 
improvement that was 26 points greater when VT was added to ST. Three months after the completion of treatment, these improvements were even greater - 30 points - for the SVT group. Several authors have estimated a significant improvement of 15 points when patients perform exercises aimed specifically at the scapula and rotator cuff stabilisers compared to nonspecific exercises (Holmgren et al., 2012). Others have likewise observed improvements in this scale after the application of certain exercises (Lewis, 2012; Litchfield, 2013). Using the SF-12 Survey, we have demonstrated that the symptoms of IS have a negative impact on a patient's QoL, physical capacity, relationship with their surroundings, and emotional state. Again, we found that there were significant changes in most of these dimensions in the experimental group, both at the end of treatment and after the 3-month follow-up. In the control group, changes were noted in fewer parameters. The characteristics of the patients who participated in our study coincide with those described in the literature (Calis et al., 2011; Garcia et al., 2016; Hanratty et al., 2012; Holmgren et al., 2012; Lewis, 2012; Litchfield, 2013; Michener et al., 2004; Tate et al., 2010; van der Windt et al., 1996). In addition, in our study population, $52 \%$ had undergone surgery to the abdominal wall, which may have contributed to alterations in the shoulder in the medium- to long-term due to insufficiencies of the abdominal musculature. Unfortunately, it is difficult to compare our results with those of other studies on VT, which have only evaluated its efficacy in treating other pathologies in adult populations. For example, Juehring and Barber combined VT and Neuromuscular Dynamic Stabilisation therapies in chronic migraine patients, observing positive effects on the pressure regulation of the circulatory system and pain reduction (Juehring and Barber, 2011), and Henze (2005) applied VT in the treatment of multiple sclerosis, achieving improvements in gait speed and stride length. Pavlú et al. (2000) applied this type of therapy in healthy adults in order to check its kinesiologic effect by means of surface electromyography, finding changes in activation of the trunk and the four extremities (Pavlú et al., 2000). This finding coincides with the type of improvements we observed in the upper limbs of our experimental group, both on the affected and healthy side, due to the global effect of Vojta activation. Indeed, several authors have described this global activation pattern, noting improvements in respiratory frequency and total body mobility (Epple et al., 2020). Our results corroborate this activation through VT and the ability of this treatment to modify patient QoL in a way that is maintained over time.

The most common modalities of physiotherapy used in the treatment of IS include electroanalgesics, TENS, ultrasound, cryo- therapy, kinesitherapy, and exercise programmes. TENS is one of the least invasive techniques for treating pain and is the most used in our healthcare centre. Like other authors, we have argued for the positive effects achieved with TENS in reducing pain and increasing mobility (Zaniewska et al., 2012). With regard to ultrasound, most studies show that it is no more effective than a placebo in treating shoulder pain (Faber et al., 2006; Michener et al., 2004). As for kinesitherapy, the exercises produce positive short- and longterm results in pain reduction and increased functionality, albeit with varying results (Kuhn, 2009; Michener et al., 2004; Varela et al., 2013).

The limitations of our study stem from its design, which did not include a nontreatment control group or a placebo group. Since the population sample was intentional, the data is not applicable to populations assigned to healthcare areas other than that used in our study. The lack of follow-up beyond 3 months is another limitation. It must also be noted that IS encompasses different pathologies with diverse anatomopathological origins; in fact, there is no consensus on the actual diagnosis of IS (Guyette et al., 2002; Michener et al., 2004). This may lead to distinct disease progressions upon treatment, which could distort the type and magnitude of response from patients with different profiles.

In conclusion, considering the number of athletes affected by subacromial IS, the growing number of athletes over 40 years old, the lack of curative treatments, and the side effects of many pharmacological pain treatments, more research in this field is necessary. In addition, the study of methods involving noninvasive interventions to achieve symptom relief is also crucial. In this study, we have evaluated for the first time the use of VT applied to IS. This procedure has been shown to be a safe and efficient conservative alternative, as it contributes to improving patient $\mathrm{QoL}$ with significant results that are superior to those of standard therapies, not only in the reduction of bodily pain, but also in the recovery of functionality, RoM, and strength. This new possibility, which would be accessible to any physiotherapeutic facility, could have a considerable economic and social impact, considering that it sustains and even improves patient functionality over time.

\section{CONFLICT OF INTEREST}

No potential conflict of interest to this article was reported.

\section{ACKNOWLEDGMENTS}

The authors received no financial support for this article. 


\section{REFERENCES}

Bongers PM. The cost of shoulder pain at work. BMJ 2001;322:64-65.

Calis HT, Berberoglu N, Calis M. Are ultrasound, laser and exercise superior to each other in the treatment of subacromial impingement syndrome? A randomized clinical trial. Eur J Phys Rehabil Med 2011;47: 375-380.

Citrome L, Magnusson K. Paging Dr Cohen, Paging Dr Cohen... An effect size interpretation is required STAT!: visualising effect size and an interview with Kristoffer Magnusson. Int J Clin Pract 2014;68:533-534.

Constant CR, Gerber C, Emery RJ, Sojbjerg JO, Gohlke F, Boileau P. A review of the Constant score: modifications and guidelines for its use. J Shoulder Elbow Surg 2008;17:355-361.

Epple C, Maurer-Burkhard B, Lichti MC, Steiner T. Vojta therapy improves postural control in very early stroke rehabilitation: a randomised controlled pilot trial. Neurol Res Pract 2020;2:23.

Faber E, Kuiper JI, Burdorf A, Miedema HS, Verhaar JA. Treatment of impingement syndrome: a systematic review of the effects on functional limitations and return to work. J Occup Rehabil 2006;16:7-25.

Garcia I, Lobo C, Lopez E, Servan JL, Tenias JM. Comparative effectiveness of ultrasonophoresis and iontophoresis in impingement syndrome: a double-blind, randomized, placebo controlled trial. Clin Rehabil 2016:30:347-358.

Guyette TM, Bae H, Warren RF, Craig E, Wickiewicz TL. Results of arthroscopic subacromial decompression in patients with subacromial impingement and glenohumeral degenerative joint disease. J Shoulder Elbow Surg 2002;11:299-304.

Hanratty CE, McVeigh JG, Kerr DP, Basford JR, Finch MB, Pendleton A, Sim J. The effectiveness of physiotherapy exercises in subacromial impingement syndrome: a systematic review and meta-analysis. Semin Arthritis Rheum 2012;42:297-316.

Henze T. Managing specific symptoms in people with multiple sclerosis. Int MS J 2005;12:60-68.

Hervas MT, Navarro Collado MJ, Peiro S, Rodrigo Perez JL, Lopez Mateu P, Martinez Tello I. Spanish version of the DASH questionnaire. Crosscultural adaptation, reliability, validity and responsiveness. Med Clin (Barc) 2006;127:441-447.

Hjermstad MJ, Fayers PM, Haugen DF, Caraceni A, Hanks GW, Loge JH, Fainsinger R, Aass N, Kaasa S; European Palliative Care Research Collaborative (EPCRC). Studies comparing Numerical Rating Scales, Verbal Rating Scales, and Visual Analogue Scales for assessment of pain intensity in adults: a systematic literature review. J Pain Symptom Manage 2011;41:1073-1093.

Holmgren T, Bjornsson Hallgren H, Oberg B, Adolfsson L, Johansson K. Effect of specific exercise strategy on need for surgery in patients with subacromial impingement syndrome: randomised controlled study. BMJ 2012;344:e787.

Ishii D, Kenmoku T, Tazawa R, Nakawaki M, Nagura N, Muneshige K, Saito K, Takaso M. Limitation of the external glenohumeral joint rotation is associated with subacromial impingement syndrome, especially pain. JSES Int 2021;5:430-438.

Juarez-Albuixech ML, Redondo-Gonzalez O, Tello I, Collado-Vazquez S, Jimenez-Antona C. Vojta Therapy versus transcutaneous electrical nerve stimulation for lumbosciatica syndrome: a quasi-experimental pilot study. J Bodyw Mov Ther 2020;24:39-46.

Juehring DD, Barber MR. A case study utilizing Vojta/Dynamic Neuromuscular Stabilization therapy to control symptoms of a chronic migraine sufferer. J Bodyw Mov Ther 2011;15:538-541.

Kuhn JE. Exercise in the treatment of rotator cuff impingement: a systematic review and a synthesized evidence-based rehabilitation protocol. J Shoulder Elbow Surg 2009;18:138-160.

Lewis JS. A specific exercise program for patients with subacromial impingement syndrome can improve function and reduce the need for surgery. J Physiother 2012;58:127.

Litchfield R. Progressive strengthening exercises for subacromial impingement syndrome. Clin J Sport Med 2013;23:86-87.

Michener LA, Walsworth MK, Burnet EN. Effectiveness of rehabilitation for patients with subacromial impingement syndrome: a systematic review. J Hand Ther 2004;17:152-164.

Pavlú D, Véle F, Havlicková L. Electromyographic and kinesiological analysis of Vojta's therapeutic principle. Rehabil Fyz Lek 2000;7:67-75.

Phillips N. Tests for diagnosing subacromial impingement syndrome and rotator cuff disease. Shoulder Elbow 2014;6:215-221.

Schulz KF, Chalmers I, Hayes RJ, Altman DG. Empirical evidence of bias. Dimensions of methodological quality associated with estimates of treatment effects in controlled trials. JAMA 1995;273:408-412.

Tate AR, McClure PW, Young IA, Salvatori R, Michener LA. Comprehensive impairment-based exercise and manual therapy intervention for patients with subacromial impingement syndrome: a case series. J Orthop Sports Phys Ther 2010;40:474-493.

Taveggia G, Borboni A, Salvi L, Mule C, Fogliaresi S, Villafane JH, Casale R. Efficacy of robot-assisted rehabilitation for the functional recovery of the upper limb in post-stroke patients: a randomized controlled study. Eur J Phys Rehabil Med 2016;52:767-773.

van der Windt DA, Koes BW, Boeke AJ, Deville W, De Jong BA, Bouter LM. Shoulder disorders in general practice: prognostic indicators of outcome. Br J Gen Pract 1996;46:519-523.

Varela E, Valero R, Kucukdeveci AA, Oral A, Ilieva E, Berteanu M, Christodoulou N, UEMS-PRM Section Professional Practice Committee. Shoulder pain management. The role of physical and rehabilitation medi- 
cine physicians. The European perspective based on the best evidence. A paper by the UEMS-PRM Section Professional Practice Committee. Eur J Phys Rehabil Med 2013;49:743-751.

Villafane JH, Lopez-Royo MP, Herrero P, Valdes K, Cantero-Tellez R, Pedersini $\mathrm{P}$, Negrini S. Prevalence of myofascial trigger points in poststroke patients with painful shoulders: a cross-sectional study. PM R 2019;11: 1077-1082.

Zaniewska R, Okurowska-Zawada B, Kulak W, Domian K. Analysis of quality of life in patiens with low back pain after receiving transcutaneous electrical nerve stimulation (TENS). Med Pr 2012;63:295-302. 